\title{
On Darbo-Sadovskii's fixed point theorems type for abstract measures of (weak) noncompactness
}

\author{
Jesús Garcia-Falset Khalid Latrach*
}

\begin{abstract}
The aim of this paper is to discuss on some generalizations of the celebrate Darbo and Sadovskii fixed point theorems without using the maximum property. Later on, we give an existence principle for a nonlinear integral equation.
\end{abstract}

\section{Introduction}

In fixed point theory one of the most important results is due to G. Darbo [11], who in 1955 using the concept of measure of noncompactness, which was introduce in 1930 by Kuratowski [15], proved a theorem which ensures the existence of a fixed point of the so-called $k$-set contraction operators and generalizes both the classical Schauder fixed point theorem and a special variant of Banach contraction principle. Darbo's result has found many applications in linear and nonlinear analysis. Such applications are characterized, in some sense, by loss of compactness which arises in many fields. Later on, Sadovskii [18] gave, also by using measures of noncompactness, a more general fixed point result than Darbo's theorem, in this case, Sadovskii introduced the concept of condensing map. As we have just mentioned, Darbo and Sadovski's results use strongly the concept of measure of noncompactness.

\footnotetext{
${ }^{*}$ Corresponding author

Received by the editors in June 2015 - In revised form in September 2015.

Communicated by Colebunders.

2010 Mathematics Subject Classification : 47H09, 47H10, 47H30.

Key words and phrases : measure of weak noncompactness, Darbo fixed point theorem, nonlinear integral equations.
} 
Since 1930, when Kuratowski gave his definition of measure of noncompactness many articles using different axioms to define a measure of noncompactness have appeared.

On the other hand, in 1977, De Blasi [12] introduced the concept of measure of weak noncompactness and in 1981, G. Emmanuele [10] stated a fixed point result for condensing mapping with respect to the measure of weak noncompactness. It is worth to notice that in Emmanuele' fixed point theorem the weak continuity of the mapping is required.

In [16], motivated by a nonlinear equation arising in transport theory, the authors established generalizations of the Schauder and Darbo fixed point theorems for the weak topology. Such results also use the concept of the De Blasi measure of weak noncompactness and, in contrast to Emmanuele' result, those do not assume the weak continuity of the mappings. In this sense, recently, in [13], a fixed point result of Sadovskii type was obtained and the author applied such result to study the existence of solutions for a generalized nonlinear Hammerstein equation on $L^{1}$ spaces.

The aim of this paper is to discuss on the axioms of a measure of (weak) noncompactness and thus to obtain some generalizations of the above mentioned Darbo, Sadovskii, etc … fixed point theorems. In particular by using Corollary 3.4 (see below), we provide an existence principle for the following nonlinear integral equation

$$
u(t)=f(t, u(t))+\int_{0}^{t} g(s, u(s)) d s,
$$

where $X$ is a Banach space, $T>0$ and $f, g:[0, T] \times X \rightarrow X$ are given functions.

\section{Preliminaries}

Throughout this paper we assume that $X$ is a Banach space. Let $\mathcal{B}(X)$ denote the collection of all nonempty bounded subsets of $X, \mathcal{K}(X)$ and $\mathcal{W}(X)$ are the subsets of $\mathcal{B}(X)$ consisting of all compact and weakly compact subsets of $X$ respectively. As usual, for any $r>0, B_{r}$ denotes the closed ball in $X$ centered in $0_{X}$ and with radius $r$. If $M$ is a subset of $X, \operatorname{co}(M)$ denotes the convex hull of $M$ and $\bar{M}$ denotes the strong closure of $M$.

We accept the following definition of a measure of (weak) noncompactness.

Definition 2.1. Let $X$ be a Banach space and $M, M_{1}, M_{2}$ belong to $\mathcal{B}(X)$. $A$ mapping $\mu: \mathcal{B}(X) \rightarrow[0, \infty[$ is said to be a measure of (weak) noncompactness in the space $X$ if it satisfies the following conditions:

(1) The family $\operatorname{ker}(\mu)=\{M \in \mathcal{B}(X): \mu(M)=0\}$ is nonempty and $\operatorname{ker}(\mu) \subset$ $\mathcal{K}(X)(\mathcal{W}(X))$.

(2) $M_{1} \subset M_{2} \Longrightarrow \mu\left(M_{1}\right) \leq \mu\left(M_{2}\right)$,

(3) $\mu(\overline{c o}(M))=\mu(M)$, 
$\operatorname{ker}(\mu)$ is called the kernel of the measure of (weak) noncompactness $\mu$.

A consequence of the definition above is that $\mu(\operatorname{co}(M))=\mu(M)$,

$$
\mu\left(\bar{M}^{w}\right)=\mu(\bar{M})=\mu(M)
$$

where $\bar{M}^{w}$ means the weak closure of $M$ (cf. [9, p. 215]).

When $\operatorname{ker}(\mu)=\mathcal{K}(X)(\mathcal{W}(X))$ the measure of (weak) noncompactness is called full. Quite often, the measure of (weak) noncompactness also satisfies:

(4) the maximum property, this is, for every $\left.x \in X, \mu\left(M_{1} \cup\{x\}\right)\right)=\mu\left(M_{1}\right)$.

(5) $\mu\left(\lambda M_{1}+(1-\lambda) M_{2}\right) \leq \lambda \mu\left(M_{1}\right)+(1-\lambda) \mu\left(M_{2}\right)$, for $\lambda \in[0,1]$,

(6) if $\left(M_{n}\right)_{n \in \mathbb{N}}$ is a sequence of closed sets from $\mathcal{B}(X)$ such that $M_{n+1} \subset M_{n}$ for $n=0,1, \cdots$ and if $\lim _{n \rightarrow \infty} \mu\left(M_{n}\right)=0$, then $M_{\infty}=\bigcap_{n=0}^{\infty} M_{n}$ is nonempty.

It is clear that if a measure of (weak) noncompactness satisfies property (6), $M_{\infty}$ belongs to $\operatorname{ker}(\mu)$ because $M_{\infty} \subseteq M_{n}$ for each $n \in \mathbb{N}$ and $\lim _{n \rightarrow \infty} \mu\left(M_{n}\right)=0$.

A measure of (weak) noncompactness is said to be regular if it has properties (1)-(6) and it is full.

We give a list of some important examples of measures of (weak) noncompactness which arise in applications. The two first examples are regular measures of noncompactness which were introduced by Kuratowski [15] and Goldenstein et al. [14] respectively.

Set measure or Kuratowski measure of noncompactness:

Given a bounded subset $A$ of $X$,

$$
\alpha(A)=\inf \left\{r>0: A \subset \cup_{i=1}^{n} D_{i}, \operatorname{diam}\left(D_{i}\right) \leq r\right\} .
$$

And the Hausdorff measure of noncompactness (or ball measure of noncompactness):

Given a bounded subset $A$ of $X$,

$$
\chi(A)=\inf \left\{r>0: A \subset \cup_{i=1}^{n} B\left(x_{i}, r\right), x_{i} \in X\right\} .
$$

The first example of a regular measure of weak noncompactness was introduced by De Blasi [12]. He defined a measure of noncompactness $\omega: \mathcal{B}(X) \rightarrow[0, \infty[$ in the following way:

$$
\omega(M):=\inf \left\{r>0: \text { there exists } W \in \mathcal{W}(X) \text { with } M \subseteq W+B_{r}\right\},
$$

for every $M \in \mathcal{B}(X)$.

The De Blasi measure of weak noncompactness has interesting properties and plays an important role in nonlinear analysis. In particular, Appell and De Pascale in [6] proved that in $L^{1}$-spaces the map $\omega(\cdot)$ can be expressed as:

$$
\omega(M)=\limsup _{\epsilon \rightarrow 0}\left\{\sup _{\psi \in M}\left[\int_{D}\|\psi(t)\|_{\gamma} d t:|D| \leq \epsilon\right]\right\},
$$


for every bounded subset $M$ of $L^{1}(\Omega ; Y)$ where $Y$ is a finite dimensional Banach space and $|D|$ denotes the Lebesgue measure of the set $D$.

Notice that in Definition 2.1 we do not assume that $\mu$ fulfills the maximum property, thus the argument developed in [7] allows us to obtain that $\mu(M)=\operatorname{diam}(M)$ and $\mu(M)=\sup \{\|x\|: x \in M\}$ are both measure of noncompactness and measure of weak noncompactness in the sense of Definition 2.1.

Let us now mention that in [8] was introduced the following measure of noncompactness which does not have the maximum property. Let $\ell_{\infty}$ be the Banach space of all bounded sequences endowed with the standard sup norm.

The following formula gives the measure of noncompactness: Given $C$ a bounded subset of $\ell_{\infty}$ and define the function $\gamma: \mathcal{B}\left(\ell_{\infty}\right) \rightarrow[0,+\infty)$ by

$$
\gamma(C):=\limsup _{n \rightarrow \infty} \operatorname{diam}\left(C_{n}\right),
$$

where $C_{n}:=\left\{x_{n}: x=\left(x_{1}, x_{2}, \cdots\right) \in C\right\}$.

Now, we introduce a measure of weak-noncompactness which does not satisfy the maximum property.

Example 2.1. Let $(X,\|\cdot\|)$ be a nonreflexive Banach space and let $\left(X \times X,\|\cdot\|_{1}\right)$ be the product space endowed with the norm $\|(a, b)\|_{1}:=\|a\|+\|b\|$. Consider $\omega(\cdot)$ and $\operatorname{diam}(\cdot)$ the measures of weak noncompactness on $X$ given by De Blasi and by the diameter respectively. Then

$$
\mu(C):=\max \left\{\omega\left(C_{1}\right), \operatorname{diam}\left(C_{2}\right)\right\},
$$

where $C_{i}, i=1,2$ denote the natural projections of $C$, defines a measure of weak noncompactness, in the sense of Definition 2.1 , on $\left(X \times X,\|\cdot\|_{1}\right)$ with property (6) and without the maximum property.

Let us see that $\mu(\cdot)$ does not satisfy the maximum property. Indeed, it is easy to check that if $\left(x_{0}, x\right),\left(x_{0}, y\right) \in X \times X$ with $x \neq y$, then $\mu\left(\left\{\left(x_{0}, x\right)\right\}\right)=0$ and $\mu\left(\left\{\left(x_{0}, y\right)\right\}\right)=0$, but

$$
\mu\left(\left\{\left(x_{0}, x\right)\right\} \cup\left\{\left(x_{0}, y\right)\right\}\right)=\mu\left(\left\{x_{0}\right\} \times\{x, y\}\right)=\|x-y\|>0 .
$$

Definition 2.2. Let $\left(X,\|\cdot\|_{X}\right)$ be a Banach and consider $\mu(\cdot)$ a measure of (weak) noncompactness on $X$. If $C$ is a nonempty subset of $X$ and $T: C \rightarrow C$ is a mapping,

(a) Given $k \in[0,1)$, the mapping $T$ is called $(\mu)$-k-set contractive if $\mu(T(A)) \leq$ $k \mu(A)$ for all $A \in \mathcal{B}(C)$.

(b) The mapping $T$ is called $(\mu)$-condensing if $\mu(T(A))<\mu(A)$ for all bounded subset $A$ of $C$ with $\mu(A)>0$.

(c) The mapping $T$ is called nonexpansive if the inequality $\|T(x)-T(y)\| \leq$ $\|x-y\|$ holds for every $x, y \in C$. 
(d) The mapping $T$ is said to be a weak contraction if the inequality $\|T(x)-T(y)\|<\|x-y\|$ holds for every $x, y \in C$ with $x \neq y$.

The following well known theorem was proved in 1967 by Sadovskii [18], it is a generalization of Darbo's fixed point theorem [11]. We refer to [4] where the reader will find many applications of these theorems.

Theorem 2.1. Suppose that $C$ is a closed convex bounded subset of a Banach space $X$ and $T: C \rightarrow C$ a continuous and condensing mapping for some regular measure of noncompactness $\mu$ on $X$, then $T$ has a fixed point.

Notice that Darbo's theorem is a particular case of Theorem 2.1 since it only works for $k$-set contractions. In $[1,2,3]$ some generalizations of Darbo's result are obtained when the measure of noncompactness defined on $X$ is under the conditions of Definition 2.1 and it fulfills condition (6), that is, the measure does not satisfies necessarily the maximum property. Nevertheless, the proof of Sadovskii's result is strongly based in the maximum property of the measure of noncompactness. One of our goal in this paper will be to discuss about Sadovskii's result when the measure of noncompactness is under the conditions of Definition 2.1.

Concerning measures of weak noncompactness we will need the following concept: a nonlinear operator $\mathbf{J}$ from a Banach space $X$ into itself enjoys Property (A) whenever:

(A) $\left\{\begin{array}{l}\text { If }\left(x_{n}\right)_{n \in \mathbb{N}} \text { is a weakly convergent sequence in } X \text {, then } \\ \left(\mathbf{J} x_{n}\right)_{n \in \mathbb{N}} \text { has a strongly convergent subsequence in } X .\end{array}\right.$

Remark 2.1. 1. Operators satisfying (A) are not necessarily weakly continuous (see [5, Theorem 2.6]).

2. A map $\mathbf{J}$ satisfies (A) if and only if it maps relatively weakly compact sets into relatively compact ones.

Now, we recall the following result established in [16] for the De Blasi measure of weak noncompactness which remains valid for any abstract measure of weak noncompactness (cf. Definition 2.1).

Theorem 2.2. Let $X$ be a Banach space, $K$ a nonempty closed convex subset of $X$ and $T$ a continuous function from $K$ into itself. If $T$ satisfies $(A)$ and $T(K)$ is relatively weakly compact, then there exists $x \in K$ such that $T x=x$.

\section{Main results}

\subsection{Sadovskii's fixed point theorem}

Theorem 3.1. Let X be a Banach space and consider $\mu(\cdot)$ a measure of noncompactness on $X$. If $M$ is a closed convex subset of $X$ and $T$ is a continuous $\mu$-condensing mapping from $M$ into itself, then $T$ has at least a fixed point whenever $T(M)$ is relatively weakly compact. 
Proof. If we define $K:=\overline{c o}(T(M))$, clearly $K$ is convex and $T$-invariant. Since $T(M)$ is relatively weakly compact, Krein- Smulian theorem ensures that it is also a weakly compact subset of $M$.

Now, we consider the following family of subsets of $K$

$$
\mathcal{F}:=\{C \subseteq K: C \text { is closed, convex and } \mathrm{T}-\text { invariant }\},
$$

$\mathcal{F} \neq \varnothing$ since $K \in \mathcal{F}$ and since $K$ is a weakly compact set, Zorn's lemma guarantees that there exists $K_{0} \in \mathcal{F}$ which is minimal with respect to the inclusion.

If we may show that $K_{0}$ is compact, then Schauder's fixed point theorem allows us to achieve the result.

Suppose that $K_{0}$ fails to be compact, in such case we have that $\mu\left(K_{0}\right)>0$ and according to our hypotheses the inequality $\mu\left(T\left(K_{0}\right)\right)<\mu\left(K_{0}\right)$ should hold. Nevertheless, taking $K_{1}=\overline{c o}\left(T\left(K_{0}\right)\right)$ it is easy to see that $K_{1} \in \mathcal{F}$ and $K_{1} \subseteq K_{0}$ (here we again have to apply Krein- Smulian theorem). Now, the minimality of $K_{0}$ yields that $K_{1}=K_{0}$. Finally, applying the properties of a measure of noncompactness, we may conclude

$$
\mu\left(K_{0}\right)=\mu\left(K_{1}\right)=\mu\left(T\left(K_{0}\right)\right)<\mu\left(K_{0}\right),
$$

which is a contradiction.

Remark 3.1. If $X$ is a reflexive Banach space, Theorem 3.1 allows us to recapture Sadovskii's theorem without using the maximum property with respect to the measure of noncompactness. However, when $X$ is nonreflexive, we only have the result if the domain of the operator, in some sense, can be included in a weakly compact set.

If in the above theorem we consider the measure of noncompactness diam $(\cdot)$, then we also obtain the uniqueness of the fixed point since in this particular case, the condition $\operatorname{diam}(T(S))<\operatorname{diam} \mu(S)$ for all $S \subset K$ with $\operatorname{diam}(S)>0$ implies that $T$ is in fact a weak contraction (i.e., $\|T x-T y\|<\|x-y\|$ ). It is interesting to notice that the existence of fixed points for a weak contractive self-mapping defined on a weakly compact convex subset of a Banach space is still an open question (see [19]). In this sense, Theorem 3.1 solves the problem for those weak contractions which are condensing with respect to the measure given by the diameter. Next example shows that such condensing mappings are not necessarily contractions.

Example 3.1. Let $\left(\ell_{1},\|\cdot\|_{1}\right)$ be the classical Banach space consisting of all real sequences $\left(x_{n}\right)$ such that $\sum_{n=1}^{\infty}\left|x_{n}\right|<\infty$ endowed with its usual norm $\left\|\left(x_{n}\right)\right\|_{1}:=$ $\sum_{n=1}^{\infty}\left|x_{n}\right|$. Let $\left(e_{n}\right)_{n \geq 1}$ be the standard Schauder basis in $\ell_{1}$, so an element $\left(x_{n}\right) \in \ell_{1}$ can be written as $\left(x_{n}\right)=\sum_{i=1}^{\infty} x_{i} e_{i}$.

Consider $K:=\left\{\left(x_{i}\right) \in B_{\ell_{1}}^{+}\right.$such that $\left.x_{1}=\frac{1}{2}\right\}$, and let $T: K \rightarrow K$ be the mapping defined by

$$
T\left(\sum_{i=1}^{\infty} x_{i} e_{i}\right)=\frac{1}{2} e_{1}+x_{2}^{2} e_{2}+\frac{1}{2} \sum_{i=3}^{\infty} x_{i} e_{i} .
$$

It is clear that $T$ is a weak contraction which is not a contraction. Moreover, it is not hard to show that $T$ is condensing with respect to the diameter measure of noncompactness. 
Theorem 3.1 along with the definition of measure of noncompactness given in (2.2) provides the following result.

Corollary 3.1. Let $\left(\ell_{\infty},\|\cdot\|_{\infty}\right)$ be the classical Banach space of all bounded sequences and let $K$ be a convex weakly compact subset of $\ell_{\infty}$. If $T: K \rightarrow K$ is a $\gamma$-condensing continuous mapping, then $T$ has at least a fixed point in $K$

Notice that in the case of the above corollary, we cannot guarantee the uniqueness of the fixed point. Nevertheless, since the set of fixed point of $T$ (say Fix $(T)$ ) satisfies that $T(\operatorname{Fix}(T))=\operatorname{Fix}(T)$, then $\gamma(\operatorname{Fix}(T))=0$ that is, it is compact. Furthermore, by definition of $\gamma(\cdot)$, we can conclude that given $\varepsilon>0$ there exists $n_{0} \in \mathbb{N}$ such that if $\left(x_{n}\right)$ and $\left(y_{n}\right)$ are two arbitrary fixed point of $T,\left|x_{n}-y_{n}\right|<\varepsilon$ whenever $n \geq n_{0}$. In particular, this fact implies that, if $T$ has a fixed point $x=\left(x_{n}\right)$ such that $x_{n} \rightarrow x_{0}$ as $n \rightarrow \infty$, then the same happens for every element of $\operatorname{Fix}(T)$.

Next example shows that, in general, $\gamma$-condensing mappings need not be weak contractions.

Example 3.2. Let $K$ be a weakly compact convex subset of $\ell_{\infty}$ such that if $x=\left(x_{1}, x_{2}, \cdots, x_{n}, \cdots\right) \in K$, then $y=\left(x_{1}, \frac{1}{2} x_{2}, \cdots, \frac{1}{2} x_{n}, \cdots\right) \in K$. If we define $T: K \rightarrow K$ by

$$
T\left(x_{1}, x_{2}, \cdots, x_{n}, \cdots\right)=\left(x_{1}, \frac{1}{2} x_{2}, \cdots, \frac{1}{2} x_{n}, \cdots\right),
$$

$T$ is a continuous mapping which is not a weak contraction. On the other hand, if $C$ is a subset of $K$, we have that $\operatorname{diam}\left((T(C))_{n}\right)=\frac{1}{2} \operatorname{diam}\left(C_{n}\right)$ for any $n \geq 2$, then $\gamma(T(C))=\frac{1}{2} \gamma(C)$, which means that $T$ is $\gamma$-condensing.

Proposition 3.1. Let $X$ be a reflexive Banach space and let $K$ be a closed and convex element of $\mathcal{B}(X)$ and $T: K \rightarrow K$ a $\mu$-condensing mapping, where $\mu(M)=$ $\sup \{\|x\|: x \in M\}$. Then $0_{X}$ is the unique fixed point of $T$.

Proof. Following the steps of the proof of Theorem 3.1, we obtain the existence of a nonempty $T$-invariant closed convex subset $K_{0}$ of $K$ such that $\mu\left(K_{0}\right)=0$, which means that $K_{0}=\left\{0_{X}\right\}$ and therefore $T\left(0_{X}\right)=0_{X}$.

Let us see that $T$ does not have another fixed point. Indeed, if there exists $0_{X} \neq$ $x_{0} \in K$ which is a fixed point of $T$, then $\left\{x_{0}\right\}=\left\{T x_{0}\right\}$. Since $\mu\left(\left\{x_{0}\right\}\right)=\left\|x_{0}\right\|>0$, we obtain the following contradiction

$$
\mu\left(\left\{x_{0}\right\}\right)=\mu\left(\left\{T x_{0}\right\}\right)<\mu\left(\left\{x_{0}\right\}\right) .
$$

Next example shows the existence of non continuous mappings satisfying the conditions of the above proposition. 
Example 3.3. Consider $(X,\|\cdot\|)$ a reflexive Banach space and let $T: B_{X} \rightarrow B_{X}$ be the mapping defined by

$$
T(x)= \begin{cases}\frac{1}{2} x, & \text { if, } \quad\|x\|<\frac{1}{2} \\ \frac{1}{3} x, & \text { if, } \quad\|x\| \geq \frac{1}{2}\end{cases}
$$

This mapping cannot be continuous on the set $\left\{x \in B_{X}:\|x\|=\frac{1}{2}\right\}$. Nevertheless, it is easy to see that for every nonempty subset $S \neq\left\{0_{X}\right\}$ of $B_{X}, \mu(T(S))<\mu(S)$.

Remark 3.2. As it was mentioned in the preliminaries section, recently Aghajain et al. in $[1,2,3]$ gave a number of results which generalize Darbo's fixed point theorem, it is not difficult to check that the conditions imposed on the mappings in such theorems imply that they become condensing for some abstract measure of noncompactness, hence in the framework of reflexive Banach spaces all these results are consequence of Theorem 3.1. However, in the proof of Theorem 3.1, we do not assume that the measure of noncompactness satisfies condition (6) which is the main tool in the proofs of their results.

\subsection{Nonreflexive Banach spaces}

Let $X$ be a nonreflexive Banach space and let $\mu(\cdot)$ be a measure of non compactness on $X$. Theorem 3.1 says that, if $K$ is a convex subset of $\mathcal{W}(X)$ and $T: K \rightarrow K$ is $\mu$-condensing, then $T$ has a fixed point. This fact is one of the reason to study conditions as in $[1,2,3]$ on the mapping supposing that $X$ is a nonreflexive Banach space. Another reason is that, in nonreflexive Banach spaces, the concept of measure of weak noncompactness takes sense if we wish to obtain Darbo's fixed point type results without assuming the weak-sequentially continuity of the mapping.

Throughout this section $X$ will denote a nonreflexive Banach space while $\mu(\cdot)$ will denote an arbitrary measure of (weak) noncompactness on $X$ satisfying condition (6).

Let $F$ be the family of all functions $\phi: \mathbb{R}^{+} \rightarrow \mathbb{R}$ satisfying

$\left(F_{1}\right) \phi$ is strictly increasing, i.e., for all $x, y \in \mathbb{R}^{+}$such that $x<y$, we have $\phi(x)<\phi(y)$

$\left(F_{2}\right)$ for each sequence $\left(\alpha_{n}\right)_{n \in \mathbb{N}}$ of positive numbers, $\lim _{n \rightarrow \infty} \alpha_{n}=0$ if and only if $\lim _{n \rightarrow \infty} \phi\left(\alpha_{n}\right)=-\infty$

$\left(F_{3}\right) \phi$ is continuous in $(0,+\infty)$.

Theorem 3.2. Let $K$ be a closed convex element of $\mathcal{B}(X)$. Let $\mu$ be a measure of noncompactness satisfying condition (6) and let $T: K \rightarrow K$ be a continuous mapping. If there exist $\tau>0, \phi: \mathbb{R}^{+} \rightarrow \mathbb{R}$ satisfying condition $\left(F_{2}\right)$ such that, for every $M \subset K$ 


$$
\mu(T(M))>0 \Longrightarrow \tau+\phi(\mu(T(M))) \leq \phi(\mu(M)),
$$

then $T$ has at least a fixed point.

Proof. Let $K_{0}=K$ and define the sequence $\left(K_{n}\right)_{n \in \mathbb{N}}$ by $K_{n+1}=\overline{c o}\left(T\left(K_{n}\right)\right)$, $n=0,1, \cdots$. Note that $T\left(K_{0}\right)=T(K) \subset K=K_{0}, K_{1}=\overline{c o}\left(T\left(K_{0}\right)\right) \subset K=K_{0}$, and by induction we get

$$
K_{0} \supset K_{1} \supset K_{2} \supset \cdots .
$$

If there exists $N \in \mathbb{N}$ such that $\mu\left(T\left(K_{N}\right)\right)=0$, then $K_{N+1}$ is relatively compact. Since $T\left(K_{N+1}\right) \subset \overline{c o}\left(T\left(K_{N+1}\right)\right)=K_{N+2} \subset K_{N+1}$, applying Schauder's Theorem we infer that $T$ has a fixed point. Assume now that, for all $n \geq 0, \mu\left(T\left(K_{n}\right)\right)>0$. Using (3.1), we get

$$
\phi\left(\mu\left(K_{n+1}\right)\right)=\phi\left(\mu\left(\overline{c o}\left(T\left(K_{n}\right)\right)\right)\right)=\phi\left(\mu\left(T\left(K_{n}\right)\right)\right) \leq \phi\left(\mu\left(K_{n}\right)\right)-\tau .
$$

Following an inductive argument, from (3.2) we have

$$
\phi\left(\mu\left(K_{n+1}\right)\right) \leq \phi\left(\mu\left(K_{0}\right)\right)-(n+1) \tau .
$$

Therefore

$$
\lim _{n \rightarrow \infty} \phi\left(\mu\left(K_{n+1}\right)\right)=-\infty .
$$

Since $\phi$ satisfies condition $\left(F_{2}\right)$, it is clear that

$$
\lim _{n \rightarrow \infty} \mu\left(K_{n+1}\right)=0 .
$$

Finally, since the sequence $\left(K_{n}\right)_{n \in \mathbb{N}}$ is decreasing and $\mu$ fulfills property (6), we have that $K_{\infty}=\bigcap_{n=0}^{\infty} K_{n}$ is a nonempty closed convex $T$-invariant subset of $K$ and $\mu\left(K_{\infty}\right)=0$, i.e. $K_{\infty}$ is compact. Now applying Schauder's theorem to the operator $T: K_{\infty} \longrightarrow K_{\infty}$ gives the desired result.

Corollary 3.2. Let $K$ be a closed convex element of $\mathcal{B}(X)$. Let $\mu$ be a measure of noncompactness satisfying condition (6) and $T: K \rightarrow K$ a continuous $k$-set contraction mapping with respect to $\mu$, then $T$ has at least a fixed point.

Proof. Theorem 3.2 yields the desired result whenever we consider $\phi(t)=\ln (t)$ and $\tau=-\ln (k)$.

Proposition 3.2. Let $K$ be a closed convex element of $\mathcal{B}(X)$ and $T: K \rightarrow K a$ mapping. If there exist $\tau>0, \phi: \mathbb{R}^{+} \rightarrow \mathbb{R}$ satisfying condition $\left(F_{2}\right)$ and for all $M \subset K$ with $\operatorname{diam}(T(M))>0$ we have

$$
\tau+\phi(\operatorname{diam}(T(M))) \leq \phi(\operatorname{diam}(M)),
$$

then $T$ has a unique fixed point. 
Proof. It is well known that $\operatorname{diam}(\cdot)$ is a measure of noncompactness in the sense of Definition 2.1 and satisfying property (6). Hence, the proof of Theorem 3.2 guarantees the existence of a $T$-invariant nonempty closed convex subset $K_{\infty}$ with $\operatorname{diam}\left(K_{\infty}\right)=0$, which means that $K_{\infty}$ is a singleton and therefore $T$ has a fixed point.

Let us see the uniqueness. Indeed, in order to get a contradiction we may suppose that there exist two different fixed points $x_{0}, x_{1} \in K$, then we may define the set $M:=\left\{x_{0}, x_{1}\right\}$. In this case $\operatorname{diam}(M)=\operatorname{diam}(T(M))=\left\|x_{0}-x_{1}\right\|>0$. This yields a contradiction because

$$
\tau+\phi(\operatorname{diam}(T(M)) \leq \phi(\operatorname{diam}(M))
$$

Recently, H. Piri and P. Kumam, [17] introduced the concept of the F-contraction as follows.

Definition 3.1. Let $(X, d)$ be a metric space. A mapping $T: X \rightarrow X$ is said to be $F$-contraction if there exist $\tau>0$ and $\phi \in F$ such that, for all $x, y \in X$,

$$
d(T x, T y)>0 \Rightarrow \tau+\phi(d(T x, T y)) \leq \phi(d(x, y))
$$

Regarding the existence and uniqueness of the fixed point, Proposition 3.2 allows us to recapture [17, Theorem 2.1] in the framework of the bounded closed convex subsets of a Banach space.

Corollary 3.3. [17] Let $(X,\|\cdot\|)$ be a Banach space and let $K$ be a closed convex element of $\mathcal{B}(X)$. Let $T: K \rightarrow K$. Suppose $\phi \in F$ and there exists $\tau>0$ such that, for all $x, y \in K$,

$$
\|T x-T y\|>0 \Rightarrow \tau+\phi(\|T x-T y\|) \leq \phi(\|x-y\|) .
$$

Then $T$ has a unique fixed point $x^{*} \in K$.

Proof. First, it is clear that $T: K \rightarrow K$ is a weak contraction (i.e., $\|T x-T y\|<$ $\|x-y\|)$. So, it is continuous.

Second, since $\phi$ is strictly increasing and continuous, we easily see that $\phi(\operatorname{diam}(K))=$ $\sup \{\phi(\|x-y\|): x, y \in K\}$.

Third, if $\operatorname{diam}(T(K))>0$, then there exist $x, y \in K$ such that $\|T x-T y\|>0$ and then, we know that

$$
\tau+\phi(\|T x-T y\|) \leq \phi(\|x-y\|)
$$

This implies that

$$
\tau+\phi(\operatorname{diam}(T(K))) \leq \phi(\operatorname{diam}(K)) .
$$

Finally, in order to obtain the result, we only have to apply the above proposition. 
Corollary 3.4. Let $(X,\|\cdot\|)$ be a Banach space and let $K$ be a closed convex element of $\mathcal{B}(X)$. Let $T, G: K \rightarrow X$ be two mappings satisfying the following conditions.

(a) $(T+G)(K) \subseteq K$,

(b) $T$ is an F-contraction,

(c) $G$ is a continuous and compact operator.

Then $J:=T+G: K \rightarrow K$ has a fixed point $x^{*} \in K$.

Proof. Let $\alpha: \mathcal{B}(X) \rightarrow[0, \infty)$ be the Kuratowski measure of noncompactness. Assume that $C$ is a subset of $K$ with $0<\alpha(C)$. By the definition of Kuratowski measure of noncompactness, for each $n \in \mathbb{N}$, there exist $S_{1}, \cdots S_{m(n)}$ bounded subsets such that $C \subseteq \cup_{i=1}^{m(n)} S_{i}$ and $\operatorname{diam}\left(S_{i}\right) \leq \alpha(C)+\frac{1}{n}$. Suppose that $\alpha(T(C))>0$. Since $T(C) \subseteq \cup_{i=1}^{m(n)} T\left(S_{i}\right)$, there exists $i_{0} \in\{1,2, \cdots, m(n)\}$ such that $\alpha(T(C)) \leq$ $\operatorname{diam}\left(T\left(S_{i_{0}}\right)\right)$. Since $T$ is an $F$ contraction, there exist $\tau>0$ and $\phi \in F$ such that, for all $x, y \in X$,

$$
\|T x-T y\|>0 \Rightarrow \tau+\phi(\|T x-T y\|) \leq \phi(\|x-y\|) .
$$

Thus, arguing as in the proof of the above corollary, we infer that

$$
\phi(\alpha(T(C))) \leq \phi\left(\operatorname{diam}\left(T\left(S_{i_{0}}\right)\right)\right) \leq \phi\left(\operatorname{diam}\left(S_{i_{0}}\right)\right)-\tau \leq \phi\left(\alpha(C)+\frac{1}{n}\right)-\tau .
$$

Now, taking limits as $n \rightarrow \infty$, the continuity of $\phi$ yields

$$
\tau+\phi(\alpha(T(C))) \leq \phi(\alpha(C)) .
$$

By using the properties of $\alpha(\cdot)$ and having in mind that $G$ is a compact operator, we obtain

$$
\begin{aligned}
\tau+\phi(\alpha(J(C)) & \leq \tau+\phi(\alpha(T(C)+G(C))) \\
& \leq \tau+\phi(\alpha(T(C))+\alpha(G(C))) \\
& =\tau+\phi(\alpha(T(C))) \\
& \leq \phi(\alpha(C)) .
\end{aligned}
$$

Finally, the use of Theorem 3.2 achieves the proof.

We also have the following result for a measure of weak noncompactness.

Theorem 3.3. Let $K$ be a closed convex element of $\mathcal{B}(X)$. Let $\mu(\cdot)$ be a measure of weak noncompactness satisfying condition (6) and let $T: K \rightarrow K$ be a continuous mapping with property $(\mathrm{A})$. If there exist $\tau>0, \phi: \mathbb{R}^{+} \rightarrow \mathbb{R}$ satisfying condition $\left(F_{2}\right)$ such that, for every $M \subset K$

$$
\mu(T(M))>0 \Longrightarrow \tau+\phi(\mu(T(M))) \leq \phi(\mu(M)),
$$

then $T$ has at least a fixed point. 
Proof. Working as in the proof of Theorem 3.2 we may obtain $K_{\infty}$ a weakly compact convex T-invariant subset. Thus, the conclusion follows from Theorem 2.2.

We close this section by the following remark.

Remark 3.3. Let $X$ be a nonreflexive Banach space and let $\mu(\cdot)$ be a measure of weak noncompactness on $X$ satisfying condition (6). Let $K$ be a closed convex element of $\mathcal{B}(X)$ and let $T$ be a a continuous mapping with property $(\mathrm{A})$.

1. If $T$ fulfills either condition (2.1) or (2.2) in [2], then $T$ has at least a fixed point.

2. If $T$ fulfills condition (2.1) in [1], then $T$ has at least a fixed point.

In order to show the above items we follow the same strategy as in the original proofs replacing Schauder's fixed point theorem by Theorem 2.2.

\section{An integral equation}

Let $(X,\|\cdot\|)$ be a Banach space. In this section we will discuss the existence of a solution $u \in C(0, T ; X)$ to the following integral equation

$$
u(t)=f(t, u(t))+\int_{0}^{t} g(s, u(s)) d s
$$

where

(a) $f:[0, T] \times X \rightarrow X$ is a continuous mapping such that there exist a $\phi$ : $(0, \infty) \rightarrow(-\infty, 0)$ bijective, strictly increasing and $\tau>0$ satisfying:

$$
\|f(t, x)-f(t, y)\|>0 \Longrightarrow \tau+\phi(\|f(t, x)-f(t, y)\|) \leq \phi(\|x-y\|)
$$

for every $t \in[0, T]$,

(b) $g:[0, T] \times X \rightarrow X$ is a continuous mapping such that there exist $m, k \in$ $L^{1}\left(0, T, \mathbb{R}^{+}\right)$and an increasing continuous function $\Omega: \mathbb{R}^{+} \rightarrow \mathbb{R}^{+}$such that

$$
\|g(t, x)\| \leq m(s) \Omega(\|x\|)+k(t)
$$

for any $x \in X$ and a.e. $t \in[0, T]$. Moreover, $g(., x)$ is measurable for $x \in X$, $g(t,$.$) is continuous for a.e. t \in[0, T]$,

(c) $\liminf _{r \rightarrow \infty} \frac{\|m\|_{1} \Omega(r)}{r}<1$.

Theorem 4.1. If (a), (b), (c) hold, then there exists at least one solution of Problem (4.1). 
Proof. It is clear that we will obtain the result if we show that the mapping $J: C(0, T ; X) \rightarrow C(0, T ; X)$ defined by

$$
J u(t)=f(t, u(t))+\int_{0}^{t} g(s, u(s)) d s
$$

has a fixed point. Now, we can introduce the following two mappings:

$$
\begin{aligned}
& G: C(0, T ; X) \rightarrow C(0, T ; X) \text { by } G(u)(t)=\int_{0}^{t} g(s, u(s)) d s \text { and } \\
& T: C(0, T ; X) \rightarrow C(0, T ; X) \text { by } T(u)(t)=f(t, u(t)) .
\end{aligned}
$$

This allows us to write $J=T+G$.

By using the Ascoli's theorem, it is not difficult to see that $G$ is a compact and continuous mapping. On the other hand, since $f(\cdot, \cdot)$ is a continuous mapping, then $T$ is also a continuous operator.

Now, we are going to show that $T$ is an F-contraction. Indeed, consider $u, v \in$ $C(0, T ; X)$ with $\|T u-T v\|_{\infty}>0$, this means that there exists $t \in[0, T]$ such that $0<\|T u-T v\|_{\infty}=\|f(t, u(t))-f(t, v(t))\|$, in this case, by (a) we have

$$
\begin{aligned}
\tau+\phi\left(\|T u-T v\|_{\infty}\right) & =\tau+\phi(\|f(t, u(t))-f(t, v(t))\|) \\
& \leq \phi(\|u(t)-v(t)\|) \leq \phi\left(\|u-v\|_{\infty}\right),
\end{aligned}
$$

which means that $T$ is an $F$-contraction. Moreover, since $\phi:(0, \infty) \rightarrow(-\infty, 0)$ is bijective and strictly increasing, it is clear that

$$
\|T u-T v\|_{\infty} \leq \phi^{-1}\left(\phi\left(\|u-v\|_{\infty}\right)-\tau\right) .
$$

Let us show that there exists $M>0$ such that $\|T u\|_{\infty} \leq M$ for every $u \in C(0, T ; X)$. If $\|T u\|_{\infty} \neq 0$, bearing in mind that $\phi\left(\|u\|_{\infty}\right)<0$, inequality (4.3) yields

$$
\begin{aligned}
\|T u\|_{\infty} & \leq\|T u-T 0\|_{\infty}+\|T 0\|_{\infty} \\
& \leq \phi^{-1}\left(\phi\left(\|u\|_{\infty}\right)-\tau\right)+\|T 0\|_{\infty} \\
& \leq \phi^{-1}(-\tau)+\|T 0\|_{\infty} .
\end{aligned}
$$

Hence, putting $M:=\phi^{-1}(-\tau)+\|T(0)\|_{\infty}$, we may conclude that

$$
\|T u\|_{\infty} \leq M
$$

Finally, given $r>0$, consider $B_{r}(0)=\left\{u \in C(0, T ; X):\|u\|_{\infty} \leq r\right\}$. We will show that there exists $r_{0}>0$ such that $J\left(B_{r_{0}}(0)\right) \subseteq B_{r_{0}}(0)$. Otherwise, for every $r>0$, we can find $u_{r} \in B_{r}(0)$ with $\left\|J\left(u_{r}\right)\right\|_{\infty}>r$. This means that $\frac{1}{r}\left\|J\left(u_{r}\right)\right\|_{\infty}>1$. Therefore we can assume that $\liminf _{r \rightarrow \infty} \frac{1}{r}\left\|J\left(u_{r}\right)\right\|_{\infty} \geq 1$. Nevertheless,

$$
\begin{aligned}
\left\|J\left(u_{r}\right)(t)\right\| & \leq\left\|f\left(t, u_{r}(t)\right)\right\|+\int_{0}^{t}\left\|g\left(s, u_{r}(s)\right)\right\| d s \\
& \leq M+\int_{0}^{t}\left\|g\left(s, u_{r}(s)\right)\right\| d s \\
& \leq M+\int_{0}^{T} m(s) \Omega\left(\left\|u_{r}(s)\right\|\right) d s+\|k\|_{1} \\
& \leq M+\Omega(r)\|m\|_{1}+\|k\|_{1} .
\end{aligned}
$$


Consequently,

$$
\liminf _{r \rightarrow \infty} \frac{1}{r}\left\|J\left(u_{r}\right)\right\|_{\infty} \leq \liminf _{r \rightarrow \infty} \frac{\|m\|_{1} \Omega(r)}{r}<1 .
$$

Thus, by Corollary 3.4 we obtain the result.

Example 4.1. Consider the nonlinear integral equation

$$
u(t)=\cos \left(e^{t}\right)+\frac{|u(t)|}{(1+\tau \sqrt{|u(t)|})^{2}}+\int_{0}^{t}(s+\sin (s) \sqrt{|u(s)|}) d s .
$$

In order to show that (4.4) admits a solution in $C(0, T ; \mathbb{R})$, we are going to check that the conditions of Theorem 4.1 are satisfied. In this case, put $X:=\mathbb{R}$ and define the function $f:[0, T] \times \mathbb{R} \rightarrow \mathbb{R}$ by

$$
f(t, x)=\cos \left(e^{t}\right)+\frac{|x|}{(1+\tau \sqrt{|x|})^{2}} .
$$

This function is continuous. Moreover,

$$
|f(t, x)-f(t, y)| \leq\left|\frac{|x|}{(1+\tau \sqrt{|x|})^{2}}-\frac{|y|}{(1+\tau \sqrt{|y|})^{2}}\right| .
$$

Notice the following elemental facts:

- The function $\varphi:[0, \infty) \rightarrow[0, \infty), \varphi(t)=\frac{t}{(1+\tau \sqrt{t})^{2}}$, is strictly increasing since $\varphi^{\prime}(t)=\frac{1}{(1+\tau \sqrt{t})^{3}}>0$,

- $\varphi$ is concave since $\varphi^{\prime \prime}(t)=\frac{-3 \tau}{2 \sqrt{t}(1+\tau \sqrt{t})^{4}}<0$,

- since $\varphi(0)=0$ and $\varphi$ is concave, then $\varphi(t+s) \leq \varphi(t)+\varphi(s)$.

Without loss of generality we can suppose that $|x| \geq|y|$. Consequently,

$$
\begin{aligned}
|f(t, x)-f(t, y)| & \leq \varphi(|x|)-\varphi(|y|) \\
& \leq \varphi(|x|-|y|) \leq \varphi(|x-y|) \\
& =\frac{|x-y|}{\left(1+\tau \sqrt{|x-y|}^{2}\right.} .
\end{aligned}
$$

Take the function $\phi:(0, \infty) \rightarrow(-\infty, 0)$ given by $\phi(t)=-\frac{1}{\sqrt{t}}$. Expression $(4.5)$ says that inequality $(4.2)$ holds. Hence, $f(\cdot, \cdot)$ satisfies condition $(a)$ of Theorem 4.1 .

The function $g:[0, T] \times \mathbb{R} \rightarrow \mathbb{R}$ defined by $g(t, x)=t+\sin (t) \sqrt{|x|}$ is continuous. Taking $m(t)=t$ and $\Omega(r)=1+\sqrt{r}$ we infer that $|g(t, x)| \leq m(t) \Omega(|x|)$. This implies that $g(\cdot, \cdot)$ fulfills condition $(b)$ of Theorem 4.1 .

Condition (c) of Theorem 4.1 holds since,

$$
\lim _{r \rightarrow \infty} \frac{\|m\|_{1} \Omega(r)}{r}=\lim _{r \rightarrow \infty} \frac{T^{2}(1+\sqrt{r})}{2 r}=0
$$

Acknowledgments. The research of the first author was partially supported by MTM 2012-34847-C02-02. 


\section{References}

[1] A. Aghajani, R. Allahyari, M. Mursaleen, A generalization of Darbo's theorem with application to the solvability of systems of integral equations. J. Comput. Appl. Math., 260 (2014), 6-877.

[2] A. Aghajani, J. Banas, N. Sabzali, Some generalizations of Darbo fixed point theorem and applications, Bull. Belg. Math. Soc., 20 (2013), 345-358.

[3] A. Aghajani, M. Mursaleen A. Shole Haghighi, Fixed point theorems for MeirKeeler condensing operators via measures of noncompactness, Acta Mathematica Scientia 35B(3) (2015), 552-566.

[4] J. Appell, Measure of noncompactness, condensing operators and fixed points: An application-oriented survey, Fixed point theory, 6 (2005), 157-229.

[5] J. Appell, The superposition operator in function spaces: a survey, Expo. Math., 6 (1988) 209-270.

[6] J. Appell, E. De Pascale, Su alcuni parametri connessi con la misura di non compattezza di Hausdorff in spazi di funzioni misurabili, Boll. Unione Mat. Ital., B 3(6) (1984), 497-515.

[7] J. Banas, On measures of noncompactness in Banach spaces, Comment. Math. Univ. Carolinae, 21 (1980), 131-143.

[8] J. Banas, K. Goebel, Measure of Noncompactness in Banach Spaces, Lecture Notes in Pure ans Applied Math., Volume 60, Marcel Dekker, 1980.

[9] J. Banas, J. Rivero, On measures of weak noncompactness, Ann. Mat. Pura Appl., 151 (4) (1988), 213-224.

[10] G. Emmanuele, Measure of weak noncompactness and fixed point theorems, Bull. Math. Soc. Sci. Math. Roum., 25, (1981), 353-358.

[11] G. Darbo, Punti uniti in transformazioni a codomio non compatto, Rend. Sem. Mat. Uni. Padova, 24 (1955), 84-92.

[12] F. S. De Blasi, On a property of the unit sphere in Banach spaces. Bull. Math. Soc. Sci. Math. Roum., 21 (1977), 259-262.

[13] J. Garcia-Falset, Existence of fixed points and measures of weak noncompactness, Nonlinear Analysis, 71 (2009), 2665-2633.

[14] L. S. Goldenstein, I. T. Gohberg, A. S. Markus, Investigations of somme properties of bounded linear operators with their q-norms, Ucen. Zap. Kishinovsk., 29 (1957), 29-36.

[15] K. Kuratowski, Sur les espaces completes, Fund. Math., 15 (1930), 301-209.

[16] K. Latrach, M.A. Taoudi, A. Zeghal, Some fixed point theorems of the Schauder and the Krasnoselskii type and application to nonlinear transport equations. J. Differential Equations, 221 (2006), 256-271. 
[17] H. Piri, P. Kumam, Some fixed point theorems concerning F-contraction in complete metric spaces, Fixed Point Theory and Applications, (2014), http:www.fixedpointtheoryandapplications.com/content/2014/1/210.

[18] B. N. Sadovskii, On a fixed point principle, Funkt. Anal. , 4(2) (1967), 74-76.

[19] H. K. Xu, Diametrically contractive mappings, Bull. Austral. Math. Soc., 70 (2004), 463-468.

Universitat de València

Departament d'Anàlisi Matemàtica

Doctor Moliner, 50, 46100 Burjassot, Spain.

email :garciaf@uv.es

Université Blaise Pascal (Clermont II)

Laboratoire de Mathématiques, CNRS UMR 6620

Campus Universitaire des Cézeaux

3, place Vasarely, TSA 60026, CS 60026

63178 Aubière, Cedex, France.

email :Khalid.Latrach@math.univ-bpclermont.fr 